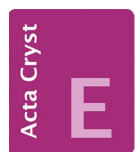

CRYSTALLOGRAPHIC COMMUNICATIONS

ISSN 2056-9890

Received 6 February 2018

Accepted 26 February 2018

Edited by K. Fejfarova, Institute of Biotechnology CAS, Czech Republic

Keywords: crystal structure; azobenzene; 2pyrrolidone; cyclic $\gamma$-aminobutyric acid derivative; GABA; racetam.

CCDC reference: 1826009

Supporting information: this article has supporting information at journals.iucr.org/e

\section{Crystal structure of 2-oxopyrrolidin-3-yl 4-(2-phenyldiazen-1-yl)benzoate}

\author{
Igor Elkin, ${ }^{\mathrm{a}}$ Thierry Maris, ${ }^{\mathrm{b}}$ Alexandre Melkoumov, ${ }^{\mathrm{c}}$ Patrice Hildgen, ${ }^{\mathrm{c}}$ Xavier \\ Banquy, ${ }^{c}$ Grégoire Leclair ${ }^{\mathrm{c}}$ and Christopher Barrett ${ }^{\mathrm{a} *}$
}

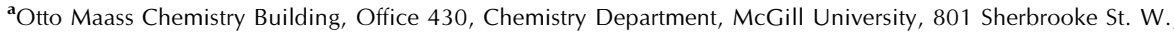
Montreal, Quebec, Canada, H3A 0B8, ${ }^{\mathbf{b}}$ Department of Chemistry, Université de Montréal, 2900 Edouard-Montpetit Blvd., Montreal, Quebec, Canada, H3C 3J7, and ${ }^{\mathrm{C}}$ Faculty of Pharmacy, Université de Montréal, 2900 Edouard-Montpetit Blvd., Montreal, Quebec, Canada, H3C 3J7. *Correspondence e-mail: chris.barrett@mcgill.ca

In the title compound, $\mathrm{C}_{17} \mathrm{H}_{15} \mathrm{~N}_{3} \mathrm{O}_{3}$, the plane of the pyrrolidone ring is inclined at an angle of $59.791(2)^{\circ}$ to that of the azobenzene segment, which adopts a configuration close to planar. In the crystal, molecules are oriented pairwise by (2-oxopyrrolidin-3-yl)oxy moieties at an angle of $76.257(3)^{\circ}$, linked by hydrogen bonds and $\pi$-stacking interactions, forming zigzag supramolecular chains parallel to [010] further linked via additional $\mathrm{C}-\mathrm{H} \cdots \pi$ interactions.

\section{Chemical context}

Cyclic derivatives of $\gamma$-aminobutyric acid, GABA, are still constituting a very promising avenue for developing new drugmolecules for improving neuronal, vascular and general cognitive functions (Malykh et al., 2010). In this context, the goal of the present study was to obtain crystals and to characterize the molecular structure of a new representative of the cyclic-GABA family (racetams), 2-oxopyrrolidin-3-yl 4-(2-phenyldiazen-1-yl)benzoate.<smiles>O=C(OC1CCNC1=O)c1ccc(/N=N/c2ccccc2)cc1</smiles>

\section{Structural commentary}

The molecular structure of the title compound (Fig. 1) comprises the expected 4-phenylazobenzoyl and (2-oxopyrrolidin-3-yl)oxy segments linked by the carboxyester bond $-\mathrm{C} 1(=\mathrm{O} 2)-\mathrm{O} 1$. The phenylazobenzoyl segment comprises two aromatic rings, one of which is linked at its para-position to the carbonyl $\mathrm{C} 8$ atom, and exhibits the more stable trans configuration to the azo group formed by $\mathrm{N} 1-\mathrm{N} 2$ atoms with a distance of 1.251 (5) $\AA$. No residual peaks are observed around the $\mathrm{N}=\mathrm{N}$ double bond as for pure azobenzene where such peaks are observed due to a dynamic pedal-like motion orientational disorder (Harada et al., 2004). The angle between the two phenyl rings is $4.29(13)^{\circ}$ and is consistent with a slight deviation of the system from the ideal planarity. Geometry parameters of the 3-oxy-substituted 2-pyrrolidone 


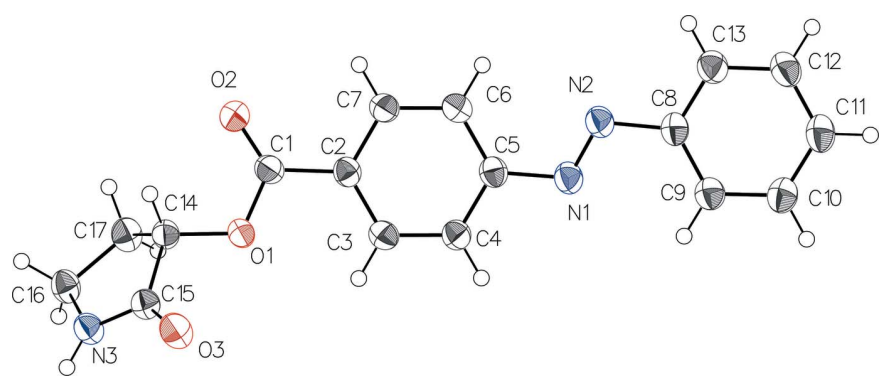

Figure 1

The molecular structure of the title compound, with displacement ellipsoids drawn at the $50 \%$ probability level. $\mathrm{H}$ atoms are shown as small spheres of arbitrary radius.

segment are close to known data (Clark et al., 2006), with a typical deviation from planarity for the non-aromatic system as shown by the torsion angles $\mathrm{C} 15-\mathrm{C} 14-\mathrm{C} 17-\mathrm{C} 16$ of $22.2(4)^{\circ}$ and $\mathrm{C} 17-\mathrm{C} 14-\mathrm{C} 15-\mathrm{N} 3$ of $-16.3(4)^{\circ}$. The Cremer $\&$ Pople puckering parameters of the five-membered ring are $Q=0.222$ (4) $\AA$ and $\varphi=279.4$ (11) ${ }^{\circ}$ conforming to an envelope on C17 (Boeyens, 1978; Cremer \& Pople, 1975).

\section{Supramolecular features}

The packing of the title molecules in the crystal (Fig. 2) is mainly determined by the presence of (2-oxopyrrolidin-3yl)oxy moieties interacting with each other pairwise, by forming hydrogen bonds between secondary amine and carbonyl groups (Table 1), similarly to other 3-oxy-substituted 2-pyrrolidone derivatives (Clark et al., 2006). This interaction
Table 1

Hydrogen-bond geometry $\left(\AA,^{\circ}\right)$.

$\mathrm{Cg} 3$ is the centrod of the $\mathrm{C} 8-\mathrm{C} 13$ ring.

\begin{tabular}{lllll}
\hline$D-\mathrm{H} \cdots A$ & $D-\mathrm{H}$ & $\mathrm{H} \cdots A$ & $D \cdots A$ & $D-\mathrm{H} \cdots A$ \\
\hline $\mathrm{N} 3-\mathrm{H} 3 A \cdots \mathrm{O}^{\mathrm{i}}$ & 0.88 & 1.99 & $2.868(4)$ & 175 \\
$\mathrm{C} 11-\mathrm{H} 11 \cdots C g 3^{\mathrm{ii}}$ & 0.95 & 2.76 & $3.596(5)$ & 147 \\
\hline
\end{tabular}

Symmetry codes: (i) $-x+\frac{3}{2}, y+\frac{1}{2},-z+1$; (ii) $-x+\frac{3}{2}, y-\frac{1}{2},-z+2$.

together with a $\pi-\pi$ interaction between the two different phenyl rings from the azobenzene moieties of adjacent molecules [distance between centroids of 3.934 (2) $\AA$ ] define a chain of corrugated molecules running along the $b$-axis direction (Fig. 2). The interactions between these chains proceed through $\mathrm{C}-\mathrm{H} \cdots \pi$ contacts involving the $\mathrm{C} 8-\mathrm{C} 13$ ring and the terminal atom $\mathrm{C} 11$ (Table 1)

\section{Database survey}

A search in the Cambridge Structural Database (Version 5.39 with one update, Groom et al., 2016) returned 101 entries for unsubstituted azobenzene, including the dynamic disorder study of Harada \& Ogawa (2004); five entries for O-paraphenylazobenzoyl monoesters (Fitjer et al., 1984; Fujino et al., 2007; Nakatsuji et al., 2007, Park et al., 2015); and only two entries for 3-oxy-substituted 2-pyrrolidone (Clark et al., 2006).

\section{Purification and crystallization}

Before recrystallization, 3-oxy(4-phenylazobenzoyl)-2-pyrrolidone was purified by the technique of flash chromatography on silica on Combi Flash Rf 150 (Teledine ISCO, Lincoln, Nebraska, USA) equipped with a SiliaSep (40 g, FLH-R10030BISO40) flash-cartridge provided by SiliCycle Inc. (Quebec, QC, Canada), using as eluent the 0$100 \%$ gradient of hexaneethyl acetate, respectively. The purity and structure of the eluate components were confirmed by the LC-MS method on an Agilent Technologies 1260 Infinity LC-MS spectrometer (Santa Clara, CA, US) in ESI positive and negative modes, equipped with an Agilent Poroshell $120 \quad$ EC-C18 $2.7 \mu \mathrm{m}$ column, using as eluent
Figure 2

A partial view of the crystal packing of the title compound. Hydrogen bonds are shown as dashed lines (see Table 1). 
Table 2

Experimental details.

\begin{tabular}{ll}
\hline Crystal data & \\
Chemical formula & $\mathrm{C}_{17} \mathrm{H}_{15} \mathrm{~N}_{3} \mathrm{O}_{3}$ \\
$M_{\mathrm{r}}$ & 309.32 \\
Crystal system, space group & Monoclinic, $C 2$ \\
Temperature (K) & 150 \\
$a, b, c(\AA)$ & $10.2069(3), 6.3761(2), 23.2265(7)$ \\
$\beta\left({ }^{\circ}\right)$ & $101.454(1)$ \\
$V\left(\AA^{3}\right)$ & $1481.48(8)$ \\
$Z$ & 4 \\
Radiation type & Ga $K \alpha, \lambda=1.34139 \AA$ \\
$\mu\left(\mathrm{mm}^{-1}\right)$ & 0.51 \\
Crystal size (mm) & $0.38 \times 0.09 \times 0.06$ \\
& \\
Data collection & Bruker Venture Metaljet \\
Diffractometer & Multi-scan $(S A D A B S ;$ Krause $e t$ \\
Absorption correction & $a l ., 2015)$ \\
& $0.433,0.581$ \\
$T_{\text {min }}, T_{\text {max }}$ & $21356,3382,3014$ \\
No. of measured, independent and & \\
$\quad$ observed $[I>2 \sigma(I)]$ reflections & 0.046 \\
$R_{\text {int }}$ & 0.650 \\
(sin $\theta / \lambda)_{\text {max }}\left(\AA^{-1}\right)$ & \\
Refinement & \\
$R\left[F^{2}>2 \sigma\left(F^{2}\right)\right], w R\left(F^{2}\right), S$ & $0.070,0.201,1.10$ \\
No. of reflections & 3382 \\
No. of parameters & 210 \\
No. of restraints & 1 \\
H-atom treatment & \\
$\Delta \rho_{\text {max }}, \Delta \rho_{\min }\left(\mathrm{e} \AA^{-3}\right)$ & $\mathrm{H}$-atom parameters constrained \\
Absolute structure & $0.43,-0.26$ \\
\hline
\end{tabular}

Computer programs: SAINT and APEX3 (Bruker, 2016), SHELXT (Sheldrick, 2015a), SHELXL2018 (Sheldrick, 2015b), OLEX2 (Dolomanov et al., 2009), Mercury (Macrae et al., 2008) and publCIF (Westrip, 2010).

the $0-100 \%$ gradient of solvent mixtures $A$ and $B$ [where $A$ : water-acetonitrile $(95 \%-5 \%)$ and acetic acid $(0.1 \%) ; B$ : acetonitrile $(100 \%)$ and acetic acid $(0.1 \%)]$ at the following conditions: a capillary voltage of ESI source of $3000 \mathrm{~V}$; a vaporizer temperature of $433 \mathrm{~K}$, a nebulization pressure of 60 psig, a dry gas temperature of $573 \mathrm{~K}$, and a gas flow of $5 \mathrm{Lmin}^{-1}$.

The crystals of the purified product were obtained by the vapor-diffusion method. A solution of $0.05 \mathrm{~g}$ of 3-oxy(4phenylazobenzoyl)-2-pyrrolidone in $1 \mathrm{~mL}$ of chloroform, in a small open container, was placed in a sealed larger container filled with hexane, above the level of the solvent, to give orange needle-shaped crystals.

\section{Refinement}

Crystal data, data collection and structure refinement details are summarized in Table 2. $\mathrm{H}$ atoms bound to $\mathrm{C}$ and $\mathrm{N}$ were positioned geometrically with $\mathrm{C}-\mathrm{H}=0.95-1.00 \AA$ and $\mathrm{N}-\mathrm{H}$ $=0.88 \AA$, and refined using a riding model with $U_{\text {iso }}(\mathrm{H})=$ $1.2 U_{\text {eq }}(\mathrm{C}$ or $\mathrm{N})$.

\section{Funding information}

Funding for this research was provided by: Natural Sciences and Engineering Research Council of Canada; Fonds de Recherche du Québec - Nature et Technologies.

\section{References}

Boeyens, J. C. A. (1978). J. Cryst. Mol. Struct. 8, 317-320.

Bruker (2016). APEX3 and SAINT. Bruker AXS Inc., Madison, Wisconsin, USA.

Clark, R. C., Pfeiffer, S. S. \& Boger, D. L. (2006). J. Am. Chem. Soc. 128, 2587-2593.

Cremer, D. \& Pople, J. A. (1975). J. Am. Chem. Soc. 97, 1354-1358.

Dolomanov, O. V., Bourhis, L. J., Gildea, R. J., Howard, J. A. K. \& Puschmann, H. (2009). J. Appl. Cryst. 42, 339-341.

Fitjer, L., Wehle, D., Noltemeyer, M., Egert, E. \& Sheldrick, G. M. (1984). Chem. Ber. 117, 203-221.

Fujino, M., Hasegawa, S., Akutsu, H., Yamada, J. \& Nakatsuji, S. (2007). Polyhedron, 26, 1989-1992.

Groom, C. R., Bruno, I. J., Lightfoot, M. P. \& Ward, S. C. (2016). Acta Cryst. B72, 171-179.

Harada, J. \& Ogawa, K. (2004). J. Am. Chem. Soc. 126, 3539-3544.

Krause, L., Herbst-Irmer, R., Sheldrick, G. M. \& Stalke, D. (2015). J. Appl. Cryst. 48, 3-10.

Macrae, C. F., Bruno, I. J., Chisholm, J. A., Edgington, P. R., McCabe, P., Pidcock, E., Rodriguez-Monge, L., Taylor, R., van de Streek, J. \& Wood, P. A. (2008). J. Appl. Cryst. 41, 466-470.

Malykh, A. G. \& Sadaie, M. R. (2010). Drugs, 70, 287-312.

Nakatsuji, S., Fujino, M., Hasegawa, S., Akutsu, H., Yamada, J., Gurman, V. S. \& Vorobiev, A. K. (2007). J. Org. Chem. 72, 20212029.

Park, J. H., Jeong, A. R., Hastuti, D. K. A. K., Jeong, M. J. \& Min, K. S. (2015). J. Incl Phenom. Macrocycl Chem. 82, 153-162.

Sheldrick, G. M. (2015a). Acta Cryst. A71, 3-8.

Sheldrick, G. M. (2015b). Acta Cryst. C71, 3-8.

Westrip, S. P. (2010). J. Appl. Cryst. 43, 920-925. 


\section{supporting information}

Acta Cryst. (2018). E74, 458-460 [https://doi.org/10.1107/S205698901800333X]

\section{Crystal structure of 2-oxopyrrolidin-3-yl 4-(2-phenyldiazen-1-yl)benzoate}

Igor Elkin, Thierry Maris, Alexandre Melkoumov, Patrice Hildgen, Xavier Banquy, Grégoire

\section{Leclair and Christopher Barrett}

\section{Computing details}

Data collection: SAINT (Bruker, 2016); cell refinement: APEX3 (Bruker, 2016); data reduction: SAINT (Bruker, 2016); program(s) used to solve structure: SHELXT (Sheldrick, 2015a); program(s) used to refine structure: SHELXL2018 (Sheldrick, 2015b); molecular graphics: OLEX2 (Dolomanov et al., 2009) and Mercury (Macrae et al., 2008); software used to prepare material for publication: OLEX2 (Dolomanov et al., 2009) and publCIF (Westrip, 2010).

2-Oxopyrrolidin-3-yl 4-(2-phenyldiazen-1-yl)benzoate

Crystal data

$\mathrm{C}_{17} \mathrm{H}_{15} \mathrm{~N}_{3} \mathrm{O}_{3}$

$M_{r}=309.32$

Monoclinic, $C 2$

$a=10.2069(3) \AA$

$b=6.3761(2) \AA$

$c=23.2265(7) \AA$

$\beta=101.454(1)^{\circ}$

$V=1481.48(8) \AA^{3}$

$Z=4$

\section{Data collection}

Bruker Venture Metaljet diffractometer

Radiation source: Metal Jet, Gallium Liquid Metal Jet Source

Helios MX Mirror Optics monochromator Detector resolution: 10.24 pixels $\mathrm{mm}^{-1}$ $\omega$ and $\varphi$ scans

Absorption correction: multi-scan

(SADABS; Krause et al., 2015)

\section{Refinement}

Refinement on $F^{2}$

Least-squares matrix: full

$R\left[F^{2}>2 \sigma\left(F^{2}\right)\right]=0.070$

$w R\left(F^{2}\right)=0.201$

$S=1.10$

3382 reflections

210 parameters

1 restraint

Primary atom site location: dual
$F(000)=648$

$D_{\mathrm{x}}=1.387 \mathrm{Mg} \mathrm{m}^{-3}$

Ga $K \alpha$ radiation, $\lambda=1.34139 \AA$

Cell parameters from 9959 reflections

$\theta=3.4-60.6^{\circ}$

$\mu=0.51 \mathrm{~mm}^{-1}$

$T=150 \mathrm{~K}$

Needle, orange

$0.38 \times 0.09 \times 0.06 \mathrm{~mm}$

$T_{\min }=0.433, T_{\max }=0.581$

21356 measured reflections

3382 independent reflections

3014 reflections with $I>2 \sigma(I)$

$R_{\text {int }}=0.046$

$\theta_{\max }=60.7^{\circ}, \theta_{\min }=3.4^{\circ}$

$h=-13 \rightarrow 13$

$k=-8 \rightarrow 8$

$l=-30 \rightarrow 30$

Hydrogen site location: inferred from neighbouring sites

$\mathrm{H}$-atom parameters constrained

$w=1 /\left[\sigma^{2}\left(F_{\mathrm{o}}^{2}\right)+(0.1386 P)^{2}+0.8495 P\right]$

where $P=\left(F_{\mathrm{o}}{ }^{2}+2 F_{\mathrm{c}}{ }^{2}\right) / 3$

$(\Delta / \sigma)_{\max }<0.001$

$\Delta \rho_{\max }=0.43 \mathrm{e} \AA^{-3}$

$\Delta \rho_{\min }=-0.26$ e $\AA^{-3}$ 
Extinction correction: (SHELXL2018; Sheldrick, 2015b),

$\mathrm{Fc}^{*}=\mathrm{kFc}\left[1+0.001 \times \mathrm{xc}^{2} \lambda^{3} / \sin (2 \theta)\right]^{-1 / 4}$
Extinction coefficient: 0.0052 (14)

Absolute structure: Refined as an inversion twin.

Special details

Experimental. X-ray crystallographic data for I were collected from a single crystal sample, which was mounted on a loop fiber. Data were collected using a Bruker Venture diffractometer equipped with a Photon 100 CMOS Detector, a Helios MX optics and a Kappa goniometer. The crystal-to-detector distance was $4.0 \mathrm{~cm}$, and the data collection was carried out in 1024 x 1024 pixel mode.

Geometry. All esds (except the esd in the dihedral angle between two 1.s. planes) are estimated using the full covariance matrix. The cell esds are taken into account individually in the estimation of esds in distances, angles and torsion angles; correlations between esds in cell parameters are only used when they are defined by crystal symmetry. An approximate (isotropic) treatment of cell esds is used for estimating esds involving l.s. planes.

Refinement. Refined as a 2-component inversion twin.

Fractional atomic coordinates and isotropic or equivalent isotropic displacement parameters $\left(\hat{A}^{2}\right)$

\begin{tabular}{|c|c|c|c|c|}
\hline & $x$ & $y$ & $z$ & $U_{\text {iso }} * / U_{\text {eq }}$ \\
\hline N1 & $0.6922(3)$ & $0.2037(6)$ & $0.83351(15)$ & $0.0367(7)$ \\
\hline $\mathrm{O} 1$ & $0.5843(2)$ & $0.9207(5)$ & $0.64242(11)$ & $0.0330(6)$ \\
\hline $\mathrm{C} 1$ & $0.4923(4)$ & $0.8542(6)$ & $0.67255(17)$ & $0.0329(8)$ \\
\hline $\mathrm{C} 2$ & $0.5463(4)$ & $0.6943(6)$ & $0.71746(16)$ & $0.0309(7)$ \\
\hline $\mathrm{O} 2$ & $0.3783(3)$ & $0.9167(6)$ & $0.66315(14)$ & $0.0473(8)$ \\
\hline N2 & $0.6075(3)$ & $0.0794(5)$ & $0.84632(15)$ & $0.0367(7)$ \\
\hline $\mathrm{C} 3$ & $0.6825(4)$ & $0.6531(6)$ & $0.73359(17)$ & $0.0337(8)$ \\
\hline H3 & 0.744646 & 0.733246 & 0.717269 & $0.040^{*}$ \\
\hline N3 & $0.6575(3)$ & $1.2842(6)$ & $0.54545(15)$ & $0.0359(7)$ \\
\hline $\mathrm{H} 3 \mathrm{~A}$ & 0.708694 & 1.324852 & 0.521220 & $0.043^{*}$ \\
\hline $\mathrm{O} 3$ & $0.6895(3)$ & $0.9284(5)$ & $0.53780(13)$ & $0.0447(7)$ \\
\hline $\mathrm{C} 4$ & $0.7272(4)$ & $0.4953(6)$ & $0.77341(17)$ & $0.0345(8)$ \\
\hline H4 & 0.820379 & 0.470969 & 0.785656 & $0.041 *$ \\
\hline $\mathrm{C} 5$ & $0.6365(4)$ & $0.3719(6)$ & $0.79569(16)$ & $0.0327(8)$ \\
\hline $\mathrm{C} 6$ & $0.4994(4)$ & $0.4147(7)$ & $0.78038(16)$ & $0.0351(8)$ \\
\hline H6 & 0.437535 & 0.333176 & 0.796487 & $0.042^{*}$ \\
\hline $\mathrm{C} 7$ & $0.4545(4)$ & $0.5760(7)$ & $0.74172(17)$ & $0.0344(8)$ \\
\hline H7 & 0.361682 & 0.606899 & 0.731581 & $0.041 *$ \\
\hline $\mathrm{C} 8$ & $0.6650(4)$ & $-0.0912(7)$ & $0.88292(16)$ & $0.0344(8)$ \\
\hline C9 & $0.8026(4)$ & $-0.1300(6)$ & $0.89767(18)$ & $0.0373(9)$ \\
\hline H9 & 0.863800 & -0.040498 & 0.883635 & $0.045^{*}$ \\
\hline $\mathrm{C} 10$ & $0.8484(4)$ & $-0.2998(7)$ & $0.93287(18)$ & $0.0411(9)$ \\
\hline H10 & 0.941490 & -0.328690 & 0.942456 & $0.049^{*}$ \\
\hline C11 & $0.7596(4)$ & $-0.4287(7)$ & $0.95435(19)$ & $0.0429(9)$ \\
\hline H11 & 0.792211 & -0.543911 & 0.978995 & $0.051^{*}$ \\
\hline $\mathrm{C} 12$ & $0.6235(5)$ & $-0.3897(7)$ & $0.93991(19)$ & $0.0421(9)$ \\
\hline H12 & 0.562923 & -0.477628 & 0.954888 & $0.051^{*}$ \\
\hline $\mathrm{C} 13$ & $0.5753(4)$ & $-0.2218(7)$ & $0.90350(19)$ & $0.0398(9)$ \\
\hline H13 & 0.481869 & -0.196631 & 0.892795 & $0.048^{*}$ \\
\hline C14 & $0.5379(4)$ & $1.0742(6)$ & $0.59755(16)$ & $0.0316(8)$ \\
\hline H14 & 0.447555 & 1.034414 & 0.574841 & $0.038^{*}$ \\
\hline
\end{tabular}




$\begin{array}{lllll}\text { C15 } & 0.6388(4) & 1.0822(6) & 0.55688(16) & 0.0324(7) \\ \text { C16 } & 0.5864(4) & 1.4325(7) & 0.57621(16) & 0.0364(8) \\ \text { H16A } & 0.511318 & 1.499253 & 0.548791 & 0.044^{*} \\ \text { H16B } & 0.647322 & 1.543044 & 0.595920 & 0.044^{*} \\ \text { C17 } & 0.5353(4) & 1.2951(6) & 0.62119(17) & 0.0380(9) \\ \text { H17A } & 0.594166 & 1.307299 & 0.660417 & 0.046^{*} \\ \text { H17B } & 0.443319 & 1.335774 & 0.624282 & 0.046^{*}\end{array}$

Atomic displacement parameters $\left(\AA^{2}\right)$

\begin{tabular}{lllllll}
\hline & $U^{11}$ & $U^{22}$ & $U^{33}$ & $U^{12}$ & $U^{13}$ & $U^{23}$ \\
\hline $\mathrm{N} 1$ & $0.0382(16)$ & $0.0309(16)$ & $0.0394(17)$ & $-0.0030(14)$ & $0.0036(13)$ & $0.0042(14)$ \\
$\mathrm{O} 1$ & $0.0343(12)$ & $0.0310(13)$ & $0.0353(12)$ & $0.0018(11)$ & $0.0110(10)$ & $0.0067(11)$ \\
$\mathrm{C} 1$ & $0.0342(17)$ & $0.0311(18)$ & $0.0353(17)$ & $-0.0006(14)$ & $0.0112(14)$ & $0.0014(14)$ \\
$\mathrm{C} 2$ & $0.0353(17)$ & $0.0255(16)$ & $0.0326(17)$ & $0.0003(15)$ & $0.0082(14)$ & $-0.0005(14)$ \\
$\mathrm{O} 2$ & $0.0379(14)$ & $0.0525(18)$ & $0.0542(17)$ & $0.0101(14)$ & $0.0154(12)$ & $0.0194(16)$ \\
$\mathrm{N} 2$ & $0.0376(16)$ & $0.0315(16)$ & $0.0401(16)$ & $-0.0028(14)$ & $0.0054(13)$ & $0.0052(14)$ \\
$\mathrm{C} 3$ & $0.0338(18)$ & $0.032(2)$ & $0.0356(18)$ & $-0.0040(14)$ & $0.0078(14)$ & $0.0005(15)$ \\
$\mathrm{N} 3$ & $0.0408(17)$ & $0.0307(16)$ & $0.0383(16)$ & $-0.0016(13)$ & $0.0124(13)$ & $0.0019(13)$ \\
O3 & $0.0593(17)$ & $0.0332(14)$ & $0.0482(15)$ & $0.0062(14)$ & $0.0266(13)$ & $0.0002(13)$ \\
C4 & $0.0340(17)$ & $0.0296(17)$ & $0.0392(19)$ & $-0.0018(15)$ & $0.0056(14)$ & $0.0019(15)$ \\
C5 & $0.0364(17)$ & $0.0278(19)$ & $0.0338(17)$ & $-0.0016(14)$ & $0.0064(14)$ & $0.0002(14)$ \\
C6 & $0.0341(17)$ & $0.0358(19)$ & $0.0364(17)$ & $-0.0029(16)$ & $0.0094(13)$ & $0.0030(17)$ \\
C7 & $0.0335(17)$ & $0.0322(19)$ & $0.0391(18)$ & $-0.0003(15)$ & $0.0109(14)$ & $0.0025(16)$ \\
C8 & $0.0401(18)$ & $0.0289(18)$ & $0.0331(17)$ & $0.0007(16)$ & $0.0045(14)$ & $0.0017(15)$ \\
C9 & $0.0396(19)$ & $0.034(2)$ & $0.0379(18)$ & $-0.0004(16)$ & $0.0071(15)$ & $0.0014(15)$ \\
C10 & $0.044(2)$ & $0.035(2)$ & $0.042(2)$ & $0.0030(17)$ & $0.0034(17)$ & $0.0014(17)$ \\
C11 & $0.052(2)$ & $0.0306(19)$ & $0.043(2)$ & $0.0007(18)$ & $0.0033(17)$ & $0.0040(17)$ \\
C12 & $0.048(2)$ & $0.035(2)$ & $0.043(2)$ & $-0.0058(18)$ & $0.0077(17)$ & $0.0048(17)$ \\
C13 & $0.041(2)$ & $0.0339(19)$ & $0.044(2)$ & $-0.0036(16)$ & $0.0051(16)$ & $0.0024(17)$ \\
C14 & $0.0337(16)$ & $0.0301(17)$ & $0.0319(16)$ & $0.0031(14)$ & $0.0087(13)$ & $0.0032(14)$ \\
C15 & $0.0356(17)$ & $0.0312(17)$ & $0.0308(16)$ & $0.0004(14)$ & $0.0080(13)$ & $-0.0004(14)$ \\
C16 & $0.0454(19)$ & $0.0282(18)$ & $0.0367(18)$ & $0.0008(17)$ & $0.0108(15)$ & $0.0007(16)$ \\
C17 & $0.048(2)$ & $0.0310(19)$ & $0.0374(19)$ & $0.0051(16)$ & $0.0149(16)$ & $-0.0018(16)$ \\
& & & & & & \\
\hline
\end{tabular}

Geometric parameters $\left(A,{ }^{\circ}\right)$

\begin{tabular}{llll}
\hline $\mathrm{N} 1-\mathrm{N} 2$ & $1.251(5)$ & $\mathrm{C} 7-\mathrm{H} 7$ & 0.9500 \\
$\mathrm{~N} 1-\mathrm{C} 5$ & $1.431(5)$ & $\mathrm{C} 8-\mathrm{C} 9$ & $1.400(5)$ \\
$\mathrm{O} 1-\mathrm{C} 1$ & $1.347(4)$ & $\mathrm{C} 8-\mathrm{C} 13$ & $1.391(6)$ \\
$\mathrm{O} 1-\mathrm{C} 14$ & $1.440(4)$ & $\mathrm{C} 9-\mathrm{H} 9$ & 0.9500 \\
$\mathrm{C} 1-\mathrm{C} 2$ & $1.484(5)$ & $\mathrm{C} 9-\mathrm{C} 10$ & $1.381(6)$ \\
$\mathrm{C} 1-\mathrm{O} 2$ & $1.208(5)$ & $\mathrm{C} 10-\mathrm{H} 10$ & 0.9500 \\
$\mathrm{C} 2-\mathrm{C} 3$ & $1.391(5)$ & $\mathrm{C} 10-\mathrm{C} 11$ & $1.387(6)$ \\
$\mathrm{C} 2-\mathrm{C} 7$ & $1.406(5)$ & $\mathrm{C} 11-\mathrm{H} 11$ & 0.9500 \\
$\mathrm{~N} 2-\mathrm{C} 8$ & $1.433(5)$ & $\mathrm{C} 11-\mathrm{C} 12$ & $1.386(7)$ \\
$\mathrm{C} 3-\mathrm{H} 3$ & 0.9500 & $\mathrm{C} 12-\mathrm{H} 12$ & 0.9500 \\
$\mathrm{C} 3-\mathrm{C} 4$ & $1.381(5)$ & $\mathrm{C} 12-\mathrm{C} 13$ & $1.392(6)$
\end{tabular}




\begin{tabular}{|c|c|c|c|}
\hline $\mathrm{N} 3-\mathrm{H} 3 \mathrm{~A}$ & 0.8800 & $\mathrm{C} 13-\mathrm{H} 13$ & 0.9500 \\
\hline $\mathrm{N} 3-\mathrm{C} 15$ & $1.336(5)$ & $\mathrm{C} 14-\mathrm{H} 14$ & 1.0000 \\
\hline $\mathrm{N} 3-\mathrm{C} 16$ & $1.462(5)$ & $\mathrm{C} 14-\mathrm{C} 15$ & $1.530(5)$ \\
\hline $\mathrm{O} 3-\mathrm{C} 15$ & $1.232(5)$ & $\mathrm{C} 14-\mathrm{C} 17$ & $1.514(5)$ \\
\hline $\mathrm{C} 4-\mathrm{H} 4$ & 0.9500 & $\mathrm{C} 16-\mathrm{H} 16 \mathrm{~A}$ & 0.9900 \\
\hline $\mathrm{C} 4-\mathrm{C} 5$ & $1.391(5)$ & C16-H16B & 0.9900 \\
\hline $\mathrm{C} 5-\mathrm{C} 6$ & $1.400(5)$ & $\mathrm{C} 16-\mathrm{C} 17$ & $1.532(5)$ \\
\hline C6- 6 6 & 0.9500 & C17-H17A & 0.9900 \\
\hline $\mathrm{C} 6-\mathrm{C} 7$ & $1.383(6)$ & C17-H17B & 0.9900 \\
\hline $\mathrm{N} 2-\mathrm{N} 1-\mathrm{C} 5$ & $114.3(3)$ & $\mathrm{C} 9-\mathrm{C} 10-\mathrm{H} 10$ & 119.7 \\
\hline $\mathrm{C} 1-\mathrm{O} 1-\mathrm{C} 14$ & $115.3(3)$ & $\mathrm{C} 9-\mathrm{C} 10-\mathrm{C} 11$ & $120.6(4)$ \\
\hline $\mathrm{O} 1-\mathrm{C} 1-\mathrm{C} 2$ & $112.4(3)$ & $\mathrm{C} 11-\mathrm{C} 10-\mathrm{H} 10$ & 119.7 \\
\hline $\mathrm{O} 2-\mathrm{C} 1-\mathrm{O} 1$ & $123.3(4)$ & $\mathrm{C} 10-\mathrm{C} 11-\mathrm{H} 11$ & 119.9 \\
\hline $\mathrm{O} 2-\mathrm{C} 1-\mathrm{C} 2$ & $124.3(4)$ & $\mathrm{C} 12-\mathrm{C} 11-\mathrm{C} 10$ & $120.1(4)$ \\
\hline $\mathrm{C} 3-\mathrm{C} 2-\mathrm{C} 1$ & $122.1(3)$ & $\mathrm{C} 12-\mathrm{C} 11-\mathrm{H} 11$ & 119.9 \\
\hline $\mathrm{C} 3-\mathrm{C} 2-\mathrm{C} 7$ & $120.1(4)$ & $\mathrm{C} 11-\mathrm{C} 12-\mathrm{H} 12$ & 120.0 \\
\hline $\mathrm{C} 7-\mathrm{C} 2-\mathrm{C} 1$ & $117.8(3)$ & $\mathrm{C} 11-\mathrm{C} 12-\mathrm{C} 13$ & $120.1(4)$ \\
\hline $\mathrm{N} 1-\mathrm{N} 2-\mathrm{C} 8$ & $113.6(3)$ & $\mathrm{C} 13-\mathrm{C} 12-\mathrm{H} 12$ & 120.0 \\
\hline $\mathrm{C} 2-\mathrm{C} 3-\mathrm{H} 3$ & 120.1 & $\mathrm{C} 8-\mathrm{C} 13-\mathrm{C} 12$ & $119.5(4)$ \\
\hline $\mathrm{C} 4-\mathrm{C} 3-\mathrm{C} 2$ & $119.8(4)$ & $\mathrm{C} 8-\mathrm{C} 13-\mathrm{H} 13$ & 120.3 \\
\hline $\mathrm{C} 4-\mathrm{C} 3-\mathrm{H} 3$ & 120.1 & $\mathrm{C} 12-\mathrm{C} 13-\mathrm{H} 13$ & 120.3 \\
\hline $\mathrm{C} 15-\mathrm{N} 3-\mathrm{H} 3 \mathrm{~A}$ & 122.5 & $\mathrm{O} 1-\mathrm{C} 14-\mathrm{H} 14$ & 110.2 \\
\hline $\mathrm{C} 15-\mathrm{N} 3-\mathrm{C} 16$ & $115.0(3)$ & $\mathrm{O} 1-\mathrm{C} 14-\mathrm{C} 15$ & $107.8(3)$ \\
\hline $\mathrm{C} 16-\mathrm{N} 3-\mathrm{H} 3 \mathrm{~A}$ & 122.5 & $\mathrm{O} 1-\mathrm{C} 14-\mathrm{C} 17$ & $113.4(3)$ \\
\hline $\mathrm{C} 3-\mathrm{C} 4-\mathrm{H} 4$ & 119.8 & $\mathrm{C} 15-\mathrm{C} 14-\mathrm{H} 14$ & 110.2 \\
\hline $\mathrm{C} 3-\mathrm{C} 4-\mathrm{C} 5$ & $120.4(4)$ & $\mathrm{C} 17-\mathrm{C} 14-\mathrm{H} 14$ & 110.2 \\
\hline $\mathrm{C} 5-\mathrm{C} 4-\mathrm{H} 4$ & 119.8 & $\mathrm{C} 17-\mathrm{C} 14-\mathrm{C} 15$ & $104.8(3)$ \\
\hline $\mathrm{C} 4-\mathrm{C} 5-\mathrm{N} 1$ & $116.1(3)$ & $\mathrm{N} 3-\mathrm{C} 15-\mathrm{C} 14$ & $107.2(3)$ \\
\hline $\mathrm{C} 4-\mathrm{C} 5-\mathrm{C} 6$ & $120.0(3)$ & $\mathrm{O} 3-\mathrm{C} 15-\mathrm{N} 3$ & $127.5(4)$ \\
\hline $\mathrm{C} 6-\mathrm{C} 5-\mathrm{N} 1$ & $123.9(3)$ & $\mathrm{O} 3-\mathrm{C} 15-\mathrm{C} 14$ & 125.3 \\
\hline $\mathrm{C} 5-\mathrm{C} 6-\mathrm{H} 6$ & 120.1 & $\mathrm{~N} 3-\mathrm{C} 16-\mathrm{H} 16 \mathrm{~A}$ & 111.1 \\
\hline $\mathrm{C} 7-\mathrm{C} 6-\mathrm{C} 5$ & $119.7(4)$ & N3-C16-H16B & 111.1 \\
\hline $\mathrm{C} 7-\mathrm{C} 6-\mathrm{H} 6$ & 120.1 & $\mathrm{~N} 3-\mathrm{C} 16-\mathrm{C} 17$ & $103.4(3)$ \\
\hline $\mathrm{C} 2-\mathrm{C} 7-\mathrm{H} 7$ & 120.1 & $\mathrm{H} 16 \mathrm{~A}-\mathrm{C} 16-\mathrm{H} 16 \mathrm{~B}$ & 109.0 \\
\hline $\mathrm{C} 6-\mathrm{C} 7-\mathrm{C} 2$ & $119.9(3)$ & $\mathrm{C} 17-\mathrm{C} 16-\mathrm{H} 16 \mathrm{~A}$ & 111.1 \\
\hline $\mathrm{C} 6-\mathrm{C} 7-\mathrm{H} 7$ & 120.1 & $\mathrm{C} 17-\mathrm{C} 16-\mathrm{H} 16 \mathrm{~B}$ & 111.1 \\
\hline $\mathrm{C} 9-\mathrm{C} 8-\mathrm{N} 2$ & 123.5 & $\mathrm{C} 14-\mathrm{C} 17-\mathrm{C} 16$ & $104.6(3)$ \\
\hline $\mathrm{C} 13-\mathrm{C} 8-\mathrm{N} 2$ & $116.0(3)$ & $\mathrm{C} 14-\mathrm{C} 17-\mathrm{H} 17 \mathrm{~A}$ & 110.8 \\
\hline $\mathrm{C} 13-\mathrm{C} 8-\mathrm{C} 9$ & $120.5(4)$ & $\mathrm{C} 14-\mathrm{C} 17-\mathrm{H} 17 \mathrm{~B}$ & 110.8 \\
\hline $\mathrm{C} 8-\mathrm{C} 9-\mathrm{H} 9$ & 120.4 & $\mathrm{C} 16-\mathrm{C} 17-\mathrm{H} 17 \mathrm{~A}$ & 110.8 \\
\hline $\mathrm{C} 10-\mathrm{C} 9-\mathrm{C} 8$ & $119.2(4)$ & $\mathrm{C} 16-\mathrm{C} 17-\mathrm{H} 17 \mathrm{~B}$ & 110.8 \\
\hline $\mathrm{C} 10-\mathrm{C} 9-\mathrm{H} 9$ & 120.4 & $\mathrm{H} 17 \mathrm{~A}-\mathrm{C} 17-\mathrm{H} 17 \mathrm{~B}$ & 108.9 \\
\hline $\mathrm{N} 1-\mathrm{N} 2-\mathrm{C} 8-\mathrm{C} 9$ & $-6.9(5)$ & $\mathrm{C} 3-\mathrm{C} 4-\mathrm{C} 5-\mathrm{C} 6$ & $-3.6(6)$ \\
\hline $\mathrm{N} 1-\mathrm{N} 2-\mathrm{C} 8-\mathrm{C} 13$ & $173.6(4)$ & $\mathrm{N} 3-\mathrm{C} 16-\mathrm{C} 17-\mathrm{C} 14$ & $-20.1(4)$ \\
\hline $\mathrm{N} 1-\mathrm{C} 5-\mathrm{C} 6-\mathrm{C} 7$ & $-177.6(4)$ & $\mathrm{C} 4-\mathrm{C} 5-\mathrm{C} 6-\mathrm{C} 7$ & $1.9(6)$ \\
\hline $\mathrm{O} 1-\mathrm{C} 1-\mathrm{C} 2-\mathrm{C} 3$ & $12.1(5)$ & $\mathrm{C} 5-\mathrm{N} 1-\mathrm{N} 2-\mathrm{C} 8$ & $178.4(3)$ \\
\hline
\end{tabular}




$\begin{array}{llll}\mathrm{O} 1-\mathrm{C} 1-\mathrm{C} 2-\mathrm{C} 7 & -164.8(3) & \mathrm{C} 5-\mathrm{C} 6-\mathrm{C} 7-\mathrm{C} 2 & 0.8(6) \\ \mathrm{O} 1-\mathrm{C} 14-\mathrm{C} 15-\mathrm{N} 3 & -137.4(3) & \mathrm{C} 7-\mathrm{C} 2-\mathrm{C} 3-\mathrm{C} 4 & 0.1(6) \\ \mathrm{O} 1-\mathrm{C} 14-\mathrm{C} 15-\mathrm{O} 3 & 44.1(5) & \mathrm{C} 8-\mathrm{C} 9-\mathrm{C} 10-\mathrm{C} 11 & -1.2(6) \\ \mathrm{O} 1-\mathrm{C} 14-\mathrm{C} 17-\mathrm{C} 16 & 139.4(3) & \mathrm{C} 9-\mathrm{C} 8-\mathrm{C} 13-\mathrm{C} 12 & 1.2(6) \\ \mathrm{C} 1-\mathrm{O} 1-\mathrm{C} 14-\mathrm{C} 15 & -163.5(3) & \mathrm{C} 9-\mathrm{C} 10-\mathrm{C} 11-\mathrm{C} 12 & 0.9(6) \\ \mathrm{C} 1-\mathrm{O} 1-\mathrm{C} 14-\mathrm{C} 17 & 81.0(4) & \mathrm{C} 10-\mathrm{C} 11-\mathrm{C} 12-\mathrm{C} 13 & 0.4(6) \\ \mathrm{C} 1-\mathrm{C} 2-\mathrm{C} 3-\mathrm{C} 4 & -176.7(3) & \mathrm{C} 11-\mathrm{C} 12-\mathrm{C} 13-\mathrm{C} 8 & -1.5(6) \\ \mathrm{C} 1-\mathrm{C} 2-\mathrm{C} 7-\mathrm{C} 6 & 175.1(4) & \mathrm{C} 13-\mathrm{C} 8-\mathrm{C} 9-\mathrm{C} 10 & 0.1(6) \\ \mathrm{C} 2-\mathrm{C} 3-\mathrm{C} 4-\mathrm{C} 5 & 2.6(6) & \mathrm{C} 14-\mathrm{O} 1-\mathrm{C} 1-\mathrm{C} 2 & 179.2(3) \\ \mathrm{O} 2-\mathrm{C} 1-\mathrm{C} 2-\mathrm{C} 3 & -168.9(4) & \mathrm{C} 14-\mathrm{O} 1-\mathrm{C} 1-\mathrm{O} 2 & 0.2(6) \\ \mathrm{O} 2-\mathrm{C} 1-\mathrm{C} 2-\mathrm{C} 7 & 14.2(6) & \mathrm{C} 15-\mathrm{N} 3-\mathrm{C} 16-\mathrm{C} 17 & 10.8(4) \\ \mathrm{N} 2-\mathrm{N} 1-\mathrm{C} 5-\mathrm{C} 4 & -170.8(3) & \mathrm{C} 15-\mathrm{C} 14-\mathrm{C} 17-\mathrm{C} 16 & 22.2(4) \\ \mathrm{N} 2-\mathrm{N} 1-\mathrm{C} 5-\mathrm{C} 6 & 8.6(5) & \mathrm{C} 16-\mathrm{N} 3-\mathrm{C} 15-\mathrm{O} 3 & -178.1(4) \\ \mathrm{N} 2-\mathrm{C} 8-\mathrm{C} 9-\mathrm{C} 10 & -179.4(4) & \mathrm{C} 16-\mathrm{N} 3-\mathrm{C} 15-\mathrm{C} 14 & 3.4(4) \\ \mathrm{N} 2-\mathrm{C} 8-\mathrm{C} 13-\mathrm{C} 12 & -179.2(4) & \mathrm{C} 17-\mathrm{C} 14-\mathrm{C} 15-\mathrm{N} 3 & -16.3(4) \\ \mathrm{C} 3-\mathrm{C} 2-\mathrm{C} 7-\mathrm{C} 6 & -1.8(6) & \mathrm{C} 17-\mathrm{C} 14-\mathrm{C} 15-\mathrm{O} 3 & 165.1(4) \\ \mathrm{C} 3-\mathrm{C} 4-\mathrm{C} 5-\mathrm{N} 1 & 175.9(3) & & \end{array}$

Hydrogen-bond geometry $\left(\AA,{ }^{\circ}\right)$

$\mathrm{Cg} 3$ is the centrod of the $\mathrm{C} 8-\mathrm{C} 13$ ring.

\begin{tabular}{lllll}
\hline$D-\mathrm{H} \cdots A$ & $D-\mathrm{H}$ & $\mathrm{H} \cdots A$ & $D \cdots A$ & $D-\mathrm{H} \cdots A$ \\
\hline $\mathrm{N} 3-\mathrm{H} 3 A \cdots \mathrm{O} 3^{\mathrm{i}}$ & 0.88 & 1.99 & $2.868(4)$ & 175 \\
$\mathrm{C} 11-\mathrm{H} 11 \cdots C g 3^{\mathrm{ii}}$ & 0.95 & 2.76 & $3.596(5)$ & 147 \\
\hline
\end{tabular}

Symmetry codes: (i) $-x+3 / 2, y+1 / 2,-z+1$; (ii) $-x+3 / 2, y-1 / 2,-z+2$. 